\title{
Evaluation of Prognostic Factors in Anti-MDA5 Antibody-Positive Patients in Chongqing, China: A Retrospective Study
}

\author{
Jun Zhou \\ Wenhan Huang \\ Feifeng Ren \\ Lei Luo \\ Dongmei Huang \\ Lin Tang
}

Department of Rheumatology and Immunology, The Second Affiliated

Hospital of Chongqing Medical University,

Chongqing, People's Republic of China
Correspondence: Lin Tang Department of Rheumatology and Immunology, The Second Affiliated Hospital of Chongqing Medical University, 76 Linjiang Road, Chongqing, 400010 , People's Republic of China

Email tanglin1217@I63.com
Objective: The aim of our study was to elucidate the potential prognostic factors in antimelanoma differentiation-associated gene 5 (anti-MDA5)-positive patients.

Methods: We divided anti-MDA5-positive patients into death and survival groups. The differences in clinical characteristics were analyzed.

Results: A total of 56 cases were included. The death group comprised 10 (17.9\%) cases, and the survival group comprised $46(82.1 \%)$ cases. Median age of the death group was greater than the survival group, 59.50 years vs 39.25 years, $\mathrm{p}<0.05$. The death group had lower lymphocyte count and albumin and higher erythrocyte sedimentation rate, ferritin and lactate dehydrogenase initially ( $\mathrm{p}<0.05$, respectively). Ground-glass opacity on chest computed tomography was found more often in the death group $(\mathrm{p}<0.05)$, in which there was an absence of honey-combed shadow initially. The diagnosis of interstitial pneumonia with autoimmune features was higher in the death group than the survival group $(70 \% \mathrm{vs} 13 \%$, $\mathrm{p}<0.05)$. The median dose of maximum daily methylprednisolone in the death group $(160 \mathrm{mg} / \mathrm{d})$ was higher than that in the survival group $(48 \mathrm{mg} / \mathrm{d})(\mathrm{p}<0.05)$.

Conclusion: Advanced age, low lymphocyte count and albumin, and increased levels of inflammatory markers may portend poor prognosis in anti-MDA5-positive patients. Extralarge doses of glucocorticoid may have no additional benefit in these patients.

Keywords: anti-melanoma differentiation-associated gene 5 antibody, interstitial lung disease, clinical manifestation, prognosis

\section{Introduction}

The anti-melanoma differentiation-associated gene 5 (anti-MDA5) antibody has been studied extensively since it was first discovered. ${ }^{1,2}$ It is reported to be more common in East Asian cohorts and manifests as arthritis and cutaneous lesions complicated by interstitial lung disease (ILD), especially rapidly-progressive ILD (RP-ILD), which has a poor prognosis as compared with anti-MDA5 antibody-negative patients. ${ }^{3-7}$ Some studies have pointed out that increased ferritin, increased age and positivity for anti-Ro52 antibody are associated with poor prognosis in patients with RP-ILD with anti-MDA5 positivity. ${ }^{8,9}$ Treatment responses vary in these patients even when similar drugs are administered. The differences in clinical manifestations, treatment response and prognosis need to be elucidated further, in particular to distinguish between dead patients and survivors with anti-MDA5 positivity. Therefore, we aimed to find the potential prognostic risk factors in anti-MDA5-positive patients by analyzing the characteristics of death and survival groups with respect to clinical manifestations and treatments. 


\section{Methods}

\section{Patients and Sera}

This retrospective study included 56 anti-MDA5-positive patients who had complete medical history and were hospitalized in the Second Affiliated Hospital of Chongqing Medical University from September 2015 to September 2020, for the first time. The patients were divided in two groups, death group and survival group. According to the Declaration of Helsinki, written informed consent was obtained from all patients or their immediate families. Ethical approval was obtained from the Ethics Committee of the Second Affiliated Hospital of Chongqing Medical University.

For the diagnosis of polymyositis/dermatomyositis (PM/DM), we referred to the idiopathic inflammatory myopathy (IIM) criteria, ${ }^{10}$ for interstitial pneumonia with autoimmune features (IPAF), we referred to the 2015 consensus classification criteria of the European Society of Respiratory Diseases/American Thoracic Society, ${ }^{11}$ for Systemic lupus erythematosus (SLE), we referred to the 2009 classification criteria of the American College of Rheumatology (ACR), ${ }^{12}$ for primary Sjogren Syndrome (pSS), we referred to the 2012 classification criteria of $\mathrm{ACR},{ }^{13}$ for Rheumatoid Arthritis (RA), we referred to the 2009 classification criteria of ACR, ${ }^{14}$ for Systemic Sclerosis (SSc), we referred to the 2013 classification criteria of ACR/ EULAR. ${ }^{15}$ Rapidly progressive ILD was defined as a condition of worsening radiologic interstitial change with progressive dyspnea and hypoxemia within 1 month of the onset of respiratory symptoms. ${ }^{2}$

\section{Laboratory and Image Examinations}

All the laboratory examinations and image examinations considered were the initial examinations after admission to hospital.

Inflammatory markers included ferritin, erythrocyte sedimentation rate (ESR), C-reactive protein (CRP), fibrinogen (Fib), D-Dimer and lactate dehydrogenase (LDH). Routine blood examination included hemoglobin and white blood cell (WBC), lymphocyte, neutrophil, and platelet counts. Biochemical tests included albumin (Alb), creatine kinase (CK) and creatine kinase isoenzyme $\mathrm{MB}$ (CK-MB). Immune indices included immunoglobulin $\mathrm{G}$ ( $\mathrm{IgG})$, immunoglobulin M (IgM), myositis-specific autoantibodies, myositisassociated autoantibodies. The myositis-specific autoantibodies and myositis-associated autoantibodies were analyzed by immunoblot assay using an OMRMUN assay kit (EUROIMMUN, Beijing, China).

High-resolution computed tomography (HRCT) of chest was applied to all patients at the time of initial admission to hospital. The CT images were interpreted by two radiologists. If the two radiologists had different opinion, they made the decisions by consensus.

\section{Statistical Analysis}

The Mann-Whitney $U$-test was used for continuous data with non-normal distribution, and the results expressed as medians with interquartile range (25\% percentile- $75 \%$ percentile). Frequencies were evaluated with $\chi^{2}$ or Fisher's exact tests with percentage (\%), as appropriate. P-values $<0.05$ was considered to be statistically significant. All statistical analyses were performed using SPSS 23.0 software (IBM SPSS Statistics version 23; IBM Corp., Armonk, NY, USA).

\section{Results}

\section{Demographic Data, Clinical Symptoms}

A total of 56 cases were included in this study. The death group comprised $10(17.9 \%)$ cases and the survival group comprised 46 (82.1\%) cases. Gender comparison between the two groups did not reveal any statistically significant differences. The median age of onset in the death group was higher than the survival group $(59.5[51.75,63]$ years vs $39.25[49,63]$ years, $p<0.05)$. Cough was more common in the death group than the survival group (9 vs 26 , $\mathrm{p}<0.05)$. The demographic data and other information about the two groups are shown in Table 1.

\section{Laboratory Examinations}

The median values of lymphocyte count and albumin in the death group were $555 \mathrm{cell} / \mu \mathrm{L}$ and $29.7 \mathrm{~g} / \mathrm{L}$, respectively, initially, which were lower than that of the survival group, where the values were $1095 \mathrm{cell} / \mu \mathrm{L}$ and $34.30 \mathrm{~g} / \mathrm{L}$, respectively $(p<0.05)$. The values of ferritin, ESR and LDH were higher in the death group than the survival group ( $<<0.05$, respectively). There were no statistically significant differences in other routine blood examinations and biochemical tests, including CK and CK-MB. The laboratory examinations of the two groups are listed in Table 2. 
Table I Comparison of Demographic Data, Clinical Symptoms and Physical Signs Between Death and Survival Groups

\begin{tabular}{|c|c|c|c|}
\hline & Death $(n=10)$ & Survival $(n=46)$ & p-value \\
\hline Gender (female/male) & $4 / 6$ & $24 / 22$ & 0.364 \\
\hline Onset age (years), median (25\%, $75 \%)$ & $59.50(51.75,63.00)$ & $39.25(49.00,63.00)$ & $0.022 *$ \\
\hline BMI, median $(25 \%, 75 \%)$ & $23.13(19.90,24.47)$ & $22.12(19.40,24.70)$ & 0.814 \\
\hline Disease duration (months), median $(25 \%, 75 \%)$ & $2(1,5)$ & $3(1,12)$ & 0.275 \\
\hline Smoke, n (\%) & $3(30.0 \%)$ & $16(34.8 \%)$ & 0.542 \\
\hline \multicolumn{4}{|l|}{ Clinical symptom } \\
\hline Fever, n (\%) & $2(20.0 \%)$ & $8(17.4 \%)$ & 0.576 \\
\hline Cough, n (\%) & $9(90.0 \%)$ & $26(56.5 \%)$ & $0.047^{*}$ \\
\hline Expectoration, n (\%) & $7(70.0 \%)$ & $20(43.5 \%)$ & 0.120 \\
\hline Dyspnea, n (\%) & $6(60.0 \%)$ & $18(39.1 \%)$ & 0.196 \\
\hline
\end{tabular}

Note: *p-value was less than 0.05 .

Abbreviation: BMI, Body mass index.

Table 2 Comparison of Laboratory Examinations Between Death and Survival Groups

\begin{tabular}{|c|c|c|c|}
\hline & Death, Median (25\%, 75\%) & Survival, Median (25\%, 75\%) & p-value \\
\hline $\mathrm{RBC}($ cell $/ \mu \mathrm{L})$ & $3975(3685,4300)$ & $4270(3885,4585)$ & 0.168 \\
\hline Hemoglobin $(g / L)$ & $121(103,132)$ & $123(107,136)$ & 0.622 \\
\hline WBC (cell/ $\mu \mathrm{L})$ & $5795(4268,11,280)$ & $5660(4000,7495)$ & 0.448 \\
\hline PLT (cell/mL) & $194(148,24 I)$ & $184(137,227)$ & 0.797 \\
\hline Neutrophil (cell/ $\mu \mathrm{L})$ & $4890(2895,8940)$ & $3790(3023,5383)$ & 0.223 \\
\hline Lymphocyte & $555(493,920)$ & $1095(640,1480)$ & $0.018^{*}$ \\
\hline Ferritin & $1226.20(649.28,1500.00)$ & $452.35(231.30,855.00)$ & $0.02 I^{*}$ \\
\hline ESR (mm/lst hour) & $60(51,82)$ & $40(19,66)$ & $0.039 *$ \\
\hline $\mathrm{LDH}(\mathrm{U} / \mathrm{L})$ & $352(33 I, 4 I I)$ & $259(194,331)$ & $0.004^{*}$ \\
\hline $\mathrm{CRP}(\mathrm{mg} / \mathrm{L})$ & $16.16(6.37,65.99)$ & $7.40(5.00,20.85)$ & 0.158 \\
\hline $\mathrm{Fib}(\mathrm{g} / \mathrm{L})$ & $5.01(3.52,6.25)$ & $4.47(3.53,5.62)$ & 0.435 \\
\hline D-Dimer (mg/L) & $0.20(0.10,0.38)$ & $0.20(0.10,0.31)$ & 0.956 \\
\hline Albumin $(g / L)$ & $29.70(28.30,31.48)$ & $34.30(30.73,39.93)$ & $0.009 *$ \\
\hline CK $(\mathrm{U} / \mathrm{L})$ & $75.50(34.08,139.00)$ & $55.00(33.00,197.25)$ & 0.873 \\
\hline CK-MB (U/L) & $16.30(12.23,22.40)$ & II.50 (8.73, I6.35) & 0.093 \\
\hline $\lg G(g / L)$ & $12.49(8.25,15.94)$ & $13.86(11.50,17.67)$ & 0.294 \\
\hline $\lg M(g / L)$ & I.I3 $(0.79,1.52)$ & $1.47(1.15,1.96)$ & 0.102 \\
\hline
\end{tabular}

Note: *p-value was less than 0.05 .

Abbreviations: RBC, red blood cell; WBC, white blood cell; PLT, platelet; ESR, erythrocyte sedimentation rate; LDH, lactate dehydrogenase; CRP, C-reactive protein; Fib, fibrinogen; CK, creatine kinase; CK-MB, creatine kinase isoenzyme MB; lgG, immunoglobulin G; IgM, immunoglobulin $M$.

\section{Manifestations of HRCT}

All patients underwent HRCT of the chest. Interstitial pneumonia was present in all the patients in the death group, and cause of death was respiratory failure with RP-ILD. Forty-one $(89.1 \%)$ of the 46 patients in the survival group had pulmonary interstitial changes; among them, 15 (32.6\%) had RP-ILD. Among the HRCT manifestations, ground-glass opacity (GGO), pleural adhesion and inflammation in the upper lung were more often present in the death group $(\mathrm{p}<0.05)$. Honey-combed shadow did not exist in the death group.
There were no statistically significant differences in patchy shadows, reticle-like shadows and so on between the death and the survival groups. The comparisons of the HRCT manifestations between the two groups are presented in Table 3.

\section{Diagnoses and Treatment}

The diagnosis of IPAF was made in seven $(70 \%)$ cases in the death group and six (13\%) cases in the survival group $(p<0.05)$. The diagnosis of the other three patients in the death group was DM. In the survival group, RA, pSS, SLE 
Table 3 Comparison of High-Resolution Computed Tomography Manifestations Between Death and Survival Groups

\begin{tabular}{|l|l|l|l|}
\hline & $\begin{array}{l}\text { Death (n=10), } \\
\text { n (\%) }\end{array}$ & $\begin{array}{l}\text { Survival (n=46), } \\
\text { n (\%) }\end{array}$ & p-value \\
\hline Honey-combed shadow & 0 & $5(10.8 \%)$ & $/$ \\
Reticle-like shadow & $6(60.0 \%)$ & $12(26.1 \%)$ & 0.060 \\
Patchy shadows & $8(80.0 \%)$ & $24(52.2 \%)$ & 0.162 \\
GGO & $7(70.0 \%)$ & $15(32.6 \%)$ & $0.038^{*}$ \\
Pleural effusion & $3(30.0 \%)$ & $5(10.8 \%)$ & 0.143 \\
Pleural adhesion & $5(30.0 \%)$ & $7(15.2 \%)$ & $0.028^{*}$ \\
Pleural thickening & $8(80.0 \%)$ & $21(45.7 \%)$ & 0.080 \\
Lymphadenectasis of & $6(80.0 \%)$ & $15(32.6 \%)$ & 0.152 \\
mediastinum & & & \\
\hline
\end{tabular}

Note: *p-value was less than 0.05 .

Abbreviation: GGO, ground-glass opacity.

and SSc were present in five (10.9\%), three $(6.5 \%)$, one $(2.2 \%)$ and one $(2.2 \%)$ cases, respectively. For these thirteen patients with IPAF, the features of clinical domain were inflammatory arthritis (6 cases), raynaud's phenomenon ( 1 case) and mechanic hands ( 2 cases). The features of serologic domain were anti-Ro-52 (SSA) (9 cases), antiEJ (1 case) and anti-PM-Scl ( 1 case). The features of morphologic domain were unexplained pleural effusion or thickening (12 cases), unexplained pericardial effusion (1 case) and pulmonary arterial hypertension (2 cases).

With respect to treatment, the median dose of maximum daily methylprednisolone in the death group (160 mg/d) was higher than that of the survival group $(48 \mathrm{mg} / \mathrm{d})(\mathrm{p}<0.05)$ during hospitalization. The utility frequency of intravenous cyclophosphamide (IVCY) and intravenous immunoglobulin (IVIG) was higher in the death group $(\mathrm{p}<0.05)$ (Table 4$)$.

\section{Discussion}

Anti-MDA5 positivity is associated with RP-ILD and high mortality. It presents a formidable challenge to clinicians and is a serious burden to patients. Common clinical characteristics, which may be easily assessed and useful for evaluating prognosis need to be analyzed. It is wellknown that anti-MDA5-positive DM might occur at any age, but it is seen more frequently in middle-aged and elderly people. Older adults experienced higher pneumonia-related death rates compared with younger persons. ${ }^{16}$ We observed a similar pattern in our study. In this cohort, the median age of onset in the death group was higher than the survival group, thereby suggesting that the incidence of anti-MDA5-positive disease increases with age, as does the impact of anti-MDA5-positive disease on morbidity and mortality. The underlying reason may be the immune dysregulation in both innate and adaptive immune components; ${ }^{17}$ the reduction in general deterioration of organ function in a way dictates not only an individual's risk of developing disease, but also outcomes.

Ferritin is associated with outcomes of anti-MDA5associated DM; the higher the level of ferritin, the worse the prognosis. ${ }^{18}$ In this study, the death group had higher ferritin, and, at the same time, had higher ESR and LDH than the survival group. ESR and LDH are inflammatory markers analogous to ferritin and may be regarded as prognostic indicators.

Table 4 Comparison of Diagnoses and Treatments Between Death and Survival Groups

\begin{tabular}{|c|c|c|c|}
\hline & Death $(n=10)$ & Survival $(n=46)$ & p-value \\
\hline IPAF, n (\%) & 7 (70.0\%) & $6(13.0 \%)$ & $0.00 I^{*}$ \\
\hline DM, n (\%) & $3(30.0 \%)$ & $29(63.0 \%)$ & 0.080 \\
\hline RA, n (\%) & 0 & $5(10.8 \%)$ & l \\
\hline pSS, n (\%) & 0 & $3(6.5 \%)$ & l \\
\hline SLE, n (\%) & 0 & I (2.2\%) & l \\
\hline SSc, n (\%) & 0 & I (2.2\%) & l \\
\hline Other, n (\%) & 0 & $\mathrm{I}(2.2 \%)$ & I \\
\hline \multicolumn{4}{|l|}{ Treatment } \\
\hline Daily maximum MP dose $(\mathrm{mg})$, median $(25 \%, 75 \%)$ & $160(80,625)$ & $48(40,115)$ & $0.028 *$ \\
\hline Tacrolimus or cyclosporin A, $n(\%)$ & $2(20.0 \%)$ & $10(21.7 \%)$ & 0.990 \\
\hline IVCY, n (\%) & $9(90.0 \%)$ & $22(47.8 \%)$ & $0.03 I^{*}$ \\
\hline IVIG, n (\%) & $8(80.0 \%)$ & $12(26.1 \%)$ & $0.002 *$ \\
\hline
\end{tabular}

Note: *p-value was less than 0.05 .

Abbreviations: IPAF, interstitial pneumonia with autoimmune features; DM, dermatomyositis; RA, rheumatoid arthritis; pSS, primary sjogren's syndrome; SLE, systemic lupus erythematosus; SSc, systemic sclerosis; MP, methylprednisolone; IVCY, intravenous cyclophosphamide; IVIG, intravenous immunoglobulin. 
Although no statistically significant differences in BMI were seen in the two groups, hypoalbuminemia (generally defined as a serum albumin concentration $\leq 30 \mathrm{~g} / \mathrm{l}$ ) was common in the death group. Albumin reflects the nutritional state of the body. In an inflammatory and debilitating illness, albumin is consumed rapidly. Importantly, it is well established that low serum albumin levels, a common occurrence in critically ill patients, is associated with worse outcomes, including increased complications and reduced short-term and long-term survival in critically ill patients. ${ }^{19-21}$ Furthermore, glucocorticoid was used in our patients. Under the influence of glucocorticoids, anabolism decreases and catabolism increases; the albumin levels may, thus, fall further. Hence, hypoalbuminemia is a useful prognostic indicator. Scaling up nutrition and exogenous albumin infusions at an appropriate time may account for some of the beneficial effects. More research is needed to validate these findings.

The lymphocyte count was lower in the death group. In past assays, it has been demonstrated that lymphocyte count was lower in anti-MDA5-positive DM patients as compared with the anti-MDA5-negative group. ${ }^{22}$ Furthermore, in our anti-MDA5-positive patients, the lymphocyte count in the death group was lower than that in the survival group. A combination of low lymphocyte count and interstitial pneumonia was seen in patients in the death group whose cause of death was respiratory failure with RP-ILD; we speculate that the low lymphocyte levels in the peripheral blood may be due to migration of and participation by these cells in abnormal lung immunity. The greater the lymphocyte consumption in the lung, the lower the lymphocyte count in the peripheral blood, and the more serious the abnormal immunity and lesion in the lung. Therefore, lower lymphocyte levels may be a risk factor for RP-ILD and portend poor prognosis. Recently, tacrolimus and cyclosporin A have been demonstrated to be effective therapy in anti-MDA5-positive DM patients. ${ }^{23,24}$ The main function of these two drugs is to inhibit T-lymphocytes and cytokines, which are produced by T-lymphocyte. We suspect that tacrolimus or cyclosporin A could alleviate the abnormal immune response, including that in the lung, to mediate curative effects by inhibiting the dysfunction of T-lymphocytes, which may play a pivotal role in the occurrence and development of anti-MDA5-associated diseases.

On HRCT, honey-combed shadow, which presents chronic change in ILD did not exist in the death group; however, GGO, which reflected acute pulmonary inflammation and effusion, pleural adhesion and inflammation in the upper lung indicated poorer prognosis in the death group as compared with the survival group. Pulmonary interstitial disorders were the main clinical manifestations in some patients, especially in the death group. IPAF was present in seven of 10 cases in the death group; these numbers are higher than that in the survival group of six of 46 . As expected, the anti-MDA5 antibody-positive patients had a poor prognosis. ${ }^{18}$ IPAF is particularly dangerous in anti-MDA5-positive patients.

In previous study, anti-MDA5 antibodies have been shown to be detected only in DM or CADM. However, we found anti-MDA5 antibody in other definite connective tissue diseases (CTDs). No one with other definite CTDs who had concomitant anti-MDA5 positivity died, even if there was no proper statistical method. We suppose that the main clinical manifestations in other definite CTDs were the primary features of the disease; the effect of antiMDA5 antibody might be weak. The diagnosis of other CTDs, apart from IPAF and DM, may be a protective factor even in anti-MDA5-positive patients. Further studies are needed.

In our cohort, the maximum daily methylprednisolone dose was evidently higher in the death group than that in the survival group ( $160 \mathrm{mg} / \mathrm{d}$ vs $48 \mathrm{mg} / \mathrm{d}$ ). We suspect that extra-large doses of glucocorticoid may have no additional benefit in these patients. Isolated case reports suggest that methylprednisolone pulse therapy $(1000 \mathrm{mg}$ /day for 3 days) was effective in RP-ILD in anti-MDA-positive patients. ${ }^{25,26}$ Nevertheless, most studies and case reports showed failure of methylprednisolone pulse therapy with death of patients, even with the usage of tacrolimus, IVCY or IVIG at the same time for RP-ILD in anti-MDApositive patients. ${ }^{27-29}$ The effect of methylprednisolone pulse therapy on prognosis of anti-MDA-positive patients is still controversial. Studies have demonstrated that a dose of $1 \mathrm{mg} / \mathrm{kg} / \mathrm{day}$ of prednisolone may be beneficial for patients with anti-MDA5-positive DM with ILD. Therefore, based on our data and that of previous studies, we make a bold assertion that the dosage of glucocorticoids should less than $1 \mathrm{mg} / \mathrm{kg} /$ day.

In conclusion, due attention should be given to clinical and laboratory characteristics, such as advanced age, low lymphocyte count and albumin and high levels of inflammatory markers, as these factors may portend poor prognosis. The dosage of glucocorticoids needs careful consideration; in particular, the application of extra-large 
doses of GCC may have no additional benefit in antiMDA5-positive patients.

\section{Funding}

This work was supported by a grant from the National Natural Science Foundation of China (grant no. 81771738).

\section{Disclosure}

The authors report no conflicts of interest in this work.

\section{References}

1. Sato S, Hirakata M, Kuwana M, et al. Autoantibodies to a 140-kd polypeptide, CADM-140, in Japanese patients with clinically amyopathic dermatomyositis. Arthritis Rheum. 2005;52(5):1571-1576. doi:10.1002/art.21023

2. Sato S, Hoshino K, Satoh T, et al. RNA helicase encoded by melanoma differentiation-associated gene 5 is a major autoantigen in patients with clinically amyopathic dermatomyositis: association with rapidly progressive interstitial lung disease. Arthritis Rheum. 2009;60(7):2193-2200. doi:10.1002/art.24621

3. Ikeda S, Arita M, Morita M, et al. Interstitial lung disease in clinically amyopathic dermatomyositis with and without anti-MDA-5 antibody: to lump or split? BMC Pulm Med. 2015;15:159. doi:10.1186/s12890-015-0154-4

4. Huang K, Vinik O, Shojania K, et al. Clinical spectrum and therapeutics in Canadian patients with anti-melanoma differentiation-associated gene 5 (MDA5)-positive dermatomyositis: a case-based review. Rheumatol Int. 2019;39(11):1971-1981. doi:10.1007/s00296-019-04398-2

5. Gono T, Kawaguchi Y, Satoh T, et al. Clinical manifestation and prognostic factor in anti-melanoma differentiation-associated gene 5 antibody-associated interstitial lung disease as a complication of dermatomyositis. Rheumatology. 2010;49(9):1713-1719. doi:10.1093/rheumatology/keq149

6. Chen Z, Cao M, Plana MN, et al. Utility of anti-melanoma differentiation-associated gene 5 antibody measurement in identifying patients with dermatomyositis and a high risk for developing rapidly progressive interstitial lung disease: a review of the literature and a meta-analysis. Arthritis Care Res. 2013;65(8):1316-1324. doi:10.1002/acr.21985

7. Fiorentino D, Chung L, Zwerner J, Rosen A, Casciola-Rosen L. The mucocutaneous and systemic phenotype of dermatomyositis patients with antibodies to MDA5 (CADM-140): a retrospective study. J Am Acad Dermatol. 2011;65(1):25-34. doi:10.1016/j.jaad.2010.09.016

8. Abe Y, Matsushita M, Tada K, Yamaji K, Takasaki Y, Tamura N. Clinical characteristics and change in the antibody titres of patients with anti-MDA5 antibody-positive inflammatory myositis. Rheumatology. 2017;56(9):1492-1497. doi:10.1093/rheumatology/kex188

9. Huang W, Ren F, Wang Q, et al. Clinical features of thirty-two patients with anti-melanoma differentiation-associated gene 5 antibodies. Clin Exp Rheumatol. 2019;37(5):803-807.

10. Lundberg IE, Tjarnlund A, Bottai M, et al. 2017 European League Against Rheumatism/American College of Rheumatology classification criteria for adult and juvenile idiopathic inflammatory myopathies and their major subgroups. Ann Rheum Dis. 2017;76 (12):1955-1964. doi:10.1136/annrheumdis-2017-211468

11. Fischer A, Antoniou KM, Brown KK, et al. An official European Respiratory Society/American Thoracic Society research statement: interstitial pneumonia with autoimmune features. Eur Respir J. 2015;46(4):976-987. doi:10.1183/13993003.00150-2015
12. Petri M, Orbai AM, Alarcon GS, et al. Derivation and validation of the systemic lupus international collaborating clinics classification criteria for systemic lupus erythematosus. Arthritis Rheum. 2012;64 (8):2677-2686. doi:10.1002/art.34473

13. Shiboski SC, Shiboski CH, Criswell L, et al. American College of Rheumatology classification criteria for sjogren's syndrome: a datadriven, expert consensus approach in the sjogren's international collaborative clinical alliance cohort. Arthritis Care Res. 2012;64 (4):475-487. doi:10.1002/acr.21591

14. Aletaha D, Neogi T, Silman AJ, et al. 2010 rheumatoid arthritis classification criteria: an American College of Rheumatology/ European League Against Rheumatism collaborative initiative. Ann Rheum Dis. 2010;69(9):1580-1588. doi:10.1136/ard.2010.138461

15. van den Hoogen F, Khanna D, Fransen J, et al. 2013 classification criteria for systemic sclerosis: an American College of Rheumatology/European League against Rheumatism collaborative initiative. Arthritis Rheum. 2013;65(11):2737-2747. doi:10.1002/ art.38098

16. Cordoba E, Maduro G, Huynh M, Varma JK, Vora NM. Deaths from pneumonia-New York City, 1999-2015. Open Forum Infect Dis. 2018;5(2):ofy020. doi:10.1093/ofid/ofy020

17. Castle SC. Clinical relevance of age-related immune dysfunction. Clin Infect Dis. 2000;31(2):578-585. doi:10.1086/313947

18. Fujiki Y, Kotani T, Isoda K, et al. Evaluation of clinical prognostic factors for interstitial pneumonia in anti-MDA5 antibody-positive dermatomyositis patients. Mod Rheumatol. 2018;28(1):133-140. doi:10.1080/14397595.2017.1318468

19. Investigators SS, Finfer S, Bellomo R, et al. Effect of baseline serum albumin concentration on outcome of resuscitation with albumin or saline in patients in intensive care units: analysis of data from the saline versus albumin fluid evaluation (SAFE) study. BMJ. 2006;333 (7577): 1044.

20. Vincent JL, Dubois MJ, Navickis RJ, Wilkes MM. Hypoalbuminemia in acute illness: is there a rationale for intervention? A meta-analysis of cohort studies and controlled trials. Ann Surg. 2003;237 (3):319-334. doi:10.1097/01.SLA.0000055547.93484.87

21. de la Cruz KI, Bakaeen FG, Wang XL, et al. Hypoalbuminemia and long-term survival after coronary artery bypass: a propensity score analysis. Ann Thorac Surg. 2011;91(3):671-675. doi:10.1016/j. athoracsur.2010.09.004

22. Chen F, Wang D, Shu X, Nakashima R, Wang G. Anti-MDA5 antibody is associated with A/SIP and decreased T cells in peripheral blood and predicts poor prognosis of ILD in Chinese patients with dermatomyositis. Rheumatol Int. 2012;32(12):3909-3915. doi:10.1007/s00296-011-2323-y

23. Gonzalez-Moreno J, Raya-Cruz M, Losada-Lopez I, Cacheda AP, Oliver C, Colom B. Rapidly progressive interstitial lung disease due to anti-MDA5 antibodies without skin involvement: a case report and literature review. Rheumatol Int. 2018;38(7):1293-1296. doi:10.1007/s00296-018-3991-7

24. Tsuji H, Nakashima R, Hosono Y, et al. Multicenter Prospective Study of the efficacy and safety of combined immunosuppressive therapy with high-dose glucocorticoid, tacrolimus, and cyclophosphamide in interstitial lung diseases accompanied by anti-melanoma differentiation-associated gene 5-positive dermatomyositis. Arthritis Rheumatol. 2020;72(3):488-498.

25. Matsumoto H, Sato S, Fujita Y, et al. Rheumatoid arthritis complicated with anti-melanoma differentiation-associated gene 5 antibody-positive interstitial pneumonia. Intern Med. 2019;58 (5):737-742. doi:10.2169/internalmedicine.1613-18

26. Fujita Y, Fukui S, Suzuki T, et al. Anti-MDA5 antibody-positive dermatomyositis complicated by autoimmune-associated hemophagocytic syndrome that was successfully treated with immunosuppressive therapy and plasmapheresis. Intern Med. 2018;57 (23):3473-3478. doi:10.2169/internalmedicine.1121-18 
27. Koguchi-Yoshioka H, Okiyama N, Iwamoto K, et al. Intravenous immunoglobulin contributes to the control of antimelanoma differentiation-associated protein 5 antibody-associated dermatomyositis with palmar violaceous macules/papules. $\mathrm{Br} J$ Dermatol. 2017;177(5):1442-1446. doi:10.1111/bjd.15499

28. Sakamoto N, Ishimoto H, Nakashima S, et al. Clinical features of anti-MDA5 antibody-positive rapidly progressive interstitial lung disease without signs of dermatomyositis. Intern Med. 2019;58 (6):837-841. doi:10.2169/internalmedicine.1516-18
29. Tokunaga K, Hagino N. Dermatomyositis with rapidly progressive interstitial lung disease treated with rituximab: a report of 3 cases in Japan. Intern Med. 2017;56(11):1399-1403. doi:10.2169/ internalmedicine.56.7956

\section{Publish your work in this journal}

The International Journal of General Medicine is an international, peer-reviewed open-access journal that focuses on general and internal medicine, pathogenesis, epidemiology, diagnosis, monitoring and treatment protocols. The journal is characterized by the rapid reporting of reviews, original research and clinical studies across all disease areas. The manuscript management system is completely online and includes a very quick and fair peer-review system, which is all easy to use. Visit http://www.dovepress.com/ testimonials.php to read real quotes from published authors.

Submit your manuscript here: https://www.dovepress.com/international-journal-of-general-medicine-journal 